\title{
Recent Results on Cepheids in the Magellanic Clouds from the EROS Survey
}

\author{
J.B. Marquette (The EROS Collaboration) \\ Institut d'Astrophysique de Paris, 98bis Bd Arago, 75014 Paris, France
}

\begin{abstract}
.
Between October 1996 and February 1997 a specific campaign of the EROS experiment was devoted to Cepheids in central regions of the LMC and the SMC. The Period-Luminosity (PL) relations were determined in the EROS photometric system, and a significant slope change of the period-luminosity relation has been observed for the SMC fundamental mode Cepheids with periods shorter than 2 days.
\end{abstract}

\section{Introduction}

The different microlensing surveys have the ability to monitor millions of stars, therefore they are suitable to study Cepheid variables. In that way the EROS 1 collaboration has already reported the discovery of 97 Cepheids towards the bar of the LMC (Beaulieu et al. 1995), and 450 Cepheids towards the SMC (Beaulieu \& Sasselov 1996). The present paper reports the results of a dedicated Cepheid campaign performed between October 1996 and February 1997. Other topics concerning microlensing survey as well as astrophysical returns of the EROS experiment can be found in this volume (see Beaulieu, Graff et al., Milsztajn, Ripepi et al.).

\section{Observations and Data Reduction}

The observations were done using the new EROS2 experimental setup which consists of a $1 \mathrm{~m} \mathrm{~F} / 5$ Ritchey-Chretien telescope, with two $2 \times 4$ CCD mosaic cameras mounted in different focal planes. The simultaneous imaging is done in a two-color passband, 420-650 nm ("VEROS" band) and 620-920 nm ("R $R_{E R O S}$ " band). It has to be stressed that these bands are not standard. The available field of view covers a surface of $0.7 \mathrm{deg}$. (right ascension) $\times 1.4 \mathrm{deg}$. (declination) on the sky. A detailed description of the experiment is available elsewhere (Bauer 1997; Bauer et al. 1997).

Two fields per Magellanic Cloud (see Table 1) were monitored about once per night with an exposure time of 20 seconds. When possible, the four fields were imaged almost simultaneously at the same airmass. A total of $\sim 110-160$ images was obtained for each field. 
Table 1. Coordinates (J2000) of the field centres.

\begin{tabular}{lcccc}
\hline field & LMC1 & LMC2 & SMC1 & SMC2 \\
\hline$\alpha$ & 52334 & 51536 & 05154 & 04154 \\
$\delta$ & -694422 & -694422 & -733632 & -734232 \\
\hline \hline
\end{tabular}

\section{Data Analysis and Discussion}

After photometric reduction using the standard EROS 2 photometry package (Peida++, Ansari 1996), the systematic search for periodic variable stars was performed (for $P>0.5$ day), using an algorithm proposed by Scargle (1982), among 1,134,000 light curves in LMC and 504,000 in SMC. A $5^{\text {th }}$-order Fourier series was used to fit the light curves. This allow to distinguish classical (F) Cepheids from first overtone (1-OT) Cepheids as done in previous study (Beaulieu et al. 1995) : 1-OT Cepheids have a lower content of second and third harmonics than F Cepheids. A second visual inspection of Cepheid light curves was then performed for the few stars that lie in-between the F or 1-OT Cepheid samples. As a result we obtained a new EROS 2 Cepheid catalog, still under construction (Bauer et al. 1999) which consists of 590 Cepheids towards the SMC (351 F and 239 1-OT Cepheids) and 290 Cepheids towards the LMC (177 $\mathrm{F}$ and 113 1-OT Cepheids).

The PL relations for the F and 1-OT Cepheids are shown in Fig. 1. For LMC Cepheids, and SMC 1-OT Cepheids as well, the relations are compatible with a linear behaviour. In contrast, that of the SMC F Cepheids displays a change in the slope for periods smaller than 2 days, visible in both colours. The magnitude deviation for these short-period Cepheids, with respect to an extrapolation of the PL relation for longer period Cepheids, reaches $0.2-0.3 \mathrm{mag}$ at $P=1$ day. Hence, a simple linear fit to the full SMC F Cepheid sample would result in a biased slope and thus to an incorrect estimate of the distance to the SMC.

Note that the shorter period Cepheids are 1-OT objects, while one finds only F Cepheids at the longest periods. This fact is related to the physics of the pulsation itself and can be explained theoretically (for recent advances on Cepheid theory, see Bono, this volume). The comparison of the LMC and SMC F Cepheids population shows that there are no such stars in LMC for the shorter periods. This reflects the metallicity effect, e.g., the blue loops in LMC are not allowed to cross the instability strip at these shorter periods. In order to quantify the significance of the non-linearity in Fig. 1, we fit the PL relations (see Table 2) in two different ways: (1) using a standard linear regression for the full data; (2) using two straight lines that cross at a "break-period" $P_{\text {break }}$,

$$
f(x)=\alpha+\beta_{\mathrm{i}} \log \left(P / P_{\text {break }}\right)
$$

where $i$ is an index for $P<P_{\text {break }}$ (resp. $P>P_{\text {break }}$ ). We find the breakperiod to be 2.0 days for $\mathrm{F}$ Cepheids and use 1.4 day for 1-OT Cepheids from the known ratio of $\mathrm{F}$ and 1-OT Cepheid periods. It is beyond the scope of the present paper to give a firm explanation of the reported effect. Three hypotheses based 


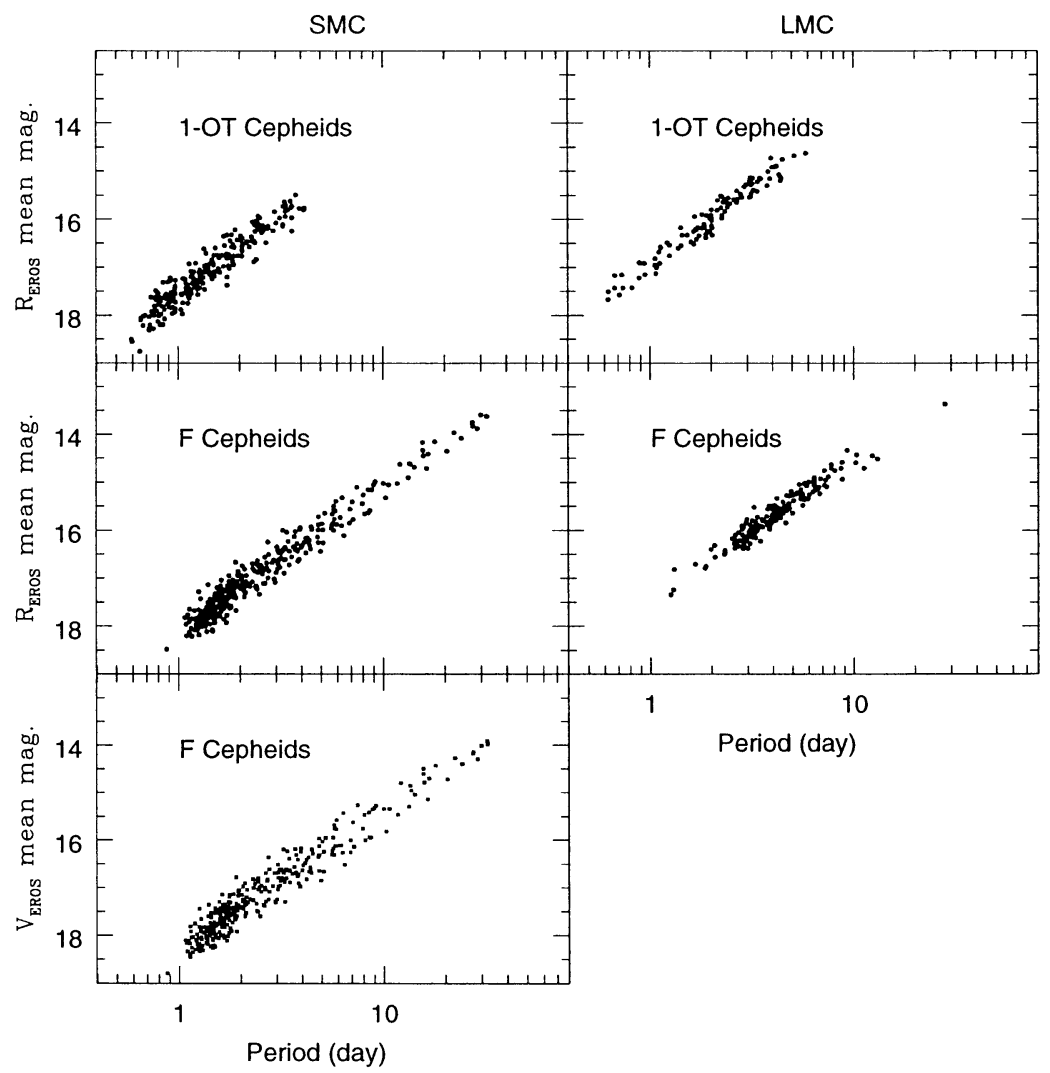

Figure 1. PL relations for first overtone Cepheids (top) and fundamental mode Cepheids (center) towards the SMC (left) and the LMC (right) seen in $R_{E R O S}$. The figure at bottom left shows the PL relation for SMC F Cepheids seen in $V_{E R O S}$. The five panels are drawn to the same scale in order to facilitate comparison.

on qualitative considerations could be proposed : (i) the observed non-linearity is due to the fact that for a given mass and metallicity the evolution tracks do not cross the entire instability strip, which would be non-uniformly populated. This is unlikely as we observe 1-OT Cepheids which would be affected in a similar way; (ii) the mixing of two stellar populations, for example, metal-poor anomalous Cepheids. This very tentative hypothesis would need further observations to be confirmed; (iii) the intrinsic shape of the instability strip becomes non-linear for small masses. The last explanation seems to be the most likely.

Acknowledgments. This work is based on observations made at ESO, La Silla, Chile. I thank the members of the EROS group for great fun, as I am back in astrophysics after six years spent in software industry. I am grateful to the IAU 190 organization committee for both invitation and travel grant. 
Table 2. PL relation fits described in Eq.(1), given in the nonstandard EROS system. $\mathrm{N}$ is the number of stars in the sample, and $\sigma_{\text {res }}$ the dispersion of the fit residuals.

\begin{tabular}{|c|c|c|c|c|c|c|}
\hline Population & Cloud & Method & $\mathrm{N}$ & $\alpha$ & $\beta$ & $\sigma_{\text {res }}$ \\
\hline \multirow{11}{*}{ F Cepheids } & \multirow{6}{*}{ LMC } & & 177 & $V_{E R O S}: 17.63 \pm 0.04$ & $2.77 \pm 0.07$ & 0.18 \\
\hline & & \multirow{3}{*}{$P>2 d$} & \multirow{3}{*}{170} & $R_{E R O S}: 17.44 \pm 0.04$ & $2.89 \pm 0.06$ & 0.15 \\
\hline & & & & $V_{E R O S}: 17.60 \pm 0.03$ & $2.72 \pm 0.07$ & 0.17 \\
\hline & & & & $R_{E R O S}: 17.43 \pm 0.02$ & $2.89 \pm 0.06$ & 0.14 \\
\hline & & \multirow[t]{2}{*}{$P<2 d$} & \multirow[t]{2}{*}{7} & $V_{E R O S}: 17.86 \pm 0.03$ & $3.59 \pm 0.57$ & 0.18 \\
\hline & & & & $R_{E R O S}: 17.46 \pm 0.02$ & $2.99 \pm 0.51$ & 0.17 \\
\hline & \multirow{5}{*}{ SMC } & \multirow[t]{2}{*}{ all } & \multirow[t]{2}{*}{351} & $V_{\text {EROS }}: 18.29 \pm 0.02$ & $2.91 \pm 0.04$ & 0.24 \\
\hline & & & & $R_{E R O S}: 18.12 \pm 0.02$ & $3.04 \pm 0.03$ & 0.20 \\
\hline & & $P>2 d$ & 164 & $V_{\text {EROS }}: 18.20 \pm 0.02$ & $\begin{array}{l}2.80 \pm 0.06 \\
2.95 \pm 0.05\end{array}$ & 0.25 \\
\hline & & \multirow{2}{*}{$P<2 d$} & \multirow{2}{*}{187} & $V_{E R O S}: 18.40 \pm 0.02$ & $3.47 \pm 0.18$ & 0.22 \\
\hline & & & & $R_{E R O S}: 18.20 \pm 0.02$ & $3.49 \pm 0.16$ & 0.20 \\
\hline \multirow{11}{*}{ 1-OT Cepheids } & \multirow{6}{*}{ LMC } & \multirow[t]{2}{*}{ all } & \multirow[t]{2}{*}{113} & $V_{E R O S}: 17.11 \pm 0.03$ & $3.12 \pm 0.07$ & 0.18 \\
\hline & & & & $R_{E R O S}: 16.93 \pm 0.03$ & $3.18 \pm 0.07$ & 0.16 \\
\hline & & \multirow{2}{*}{$P>1.4 \mathrm{~d}$} & \multirow[t]{2}{*}{87} & $V_{E R O S}: 17.13 \pm 0.03$ & $3.15 \pm 0.12$ & 0.19 \\
\hline & & & & $R_{E R O S}: 16.96 \pm 0.03$ & $3.23 \pm 0.11$ & 0.16 \\
\hline & & \multirow[t]{2}{*}{$\mathrm{P}<1.4 \mathrm{~d}$} & \multirow[t]{2}{*}{26} & $V_{E R O S}: 17.11 \pm 0.03$ & $3.03 \pm 0.21$ & 0.16 \\
\hline & & & & $R_{E R O S}: 16.93 \pm 0.03$ & $3.07 \pm 0.20$ & 0.16 \\
\hline & \multirow{5}{*}{ SMC } & \multirow{2}{*}{$P>1.4 \mathrm{~d}$} & \multirow[t]{2}{*}{239} & $V_{E R O S}: 17.70 \pm 0.02$ & $3.06 \pm 0.08$ & 0.25 \\
\hline & & & & $R_{E R O S}: 17.55 \pm 0.02$ & $3.21 \pm 0.07$ & 0.21 \\
\hline & & \multirow{3}{*}{$\mathrm{P}<1.4 \mathrm{~d}$} & 103 & $\begin{array}{l}V_{E R O S}: 17.55 \pm 0.03 \\
R_{E R O S}: 17.43 \pm 0.03\end{array}$ & $\begin{array}{l}2.71 \pm 0.26 \\
2.91 \pm 0.23\end{array}$ & $\begin{array}{l}0.25 \\
0.21\end{array}$ \\
\hline & & & 136 & $V_{E R O S}: 17.70 \pm 0.03$ & $3.20 \pm 0.12$ & 0.25 \\
\hline & & & & $R_{E R O S}: 17.55 \pm 0.03$ & $3.32 \pm, 0.1$ & 0.21 \\
\hline
\end{tabular}

\section{References}

Ansari, R. 1996, Vistas in Astronomy, 40, 519

Bauer, F. 1997, PhD thesis, Université Paris VII, or Saclay Report Dapnia-SPP $97 / 10$

Bauer, F., et al. (the EROS coll.) 1997, in Optical Detectors for Astronomy, ESO: Garching

Bauer, F., et al. (the EROS coll.) 1999, in preparation

Beaulieu, J.P., et al. (the EROS coll.) 1995, ApJ, 303, 137

Beaulieu, J.P., \& Sasselov D. (the EROS coll.) 1996, in Variable Stars and the Astrophysical Returns of Microlensing Surveys, R. Ferlet, J.P. Maillard, \& B. Raban, Frontières: Paris, 1996, 193

Scargle, J.D. 1982, ApJ, 263, 835 